H. Tanaka

Nagoya Math. J.

Vol. 38 (1970), 139-144

\title{
ON A $\Pi_{1}^{0}$ SET OF POSITIVE MEASURE
}

\author{
HISAO TANAKA
}

\section{Dedicated to Professor Katuzi Ono for his 60th birthday anniversary}

Introduction. Some basis results for arithmetic, hyperarithmetic $(H A)$ or $\Pi_{1}^{1}$ sets which have positive measure (or which are not meager, i.e., of the second Baire category) have been obtained by several authors. ${ }^{1)}$ For example, every non-meager $\Sigma_{3}^{0}$ set must have a recursive element (ShoenfieldHinman, Hinman [2]) but there exists a non-meager $\Pi_{3}^{0}$ set (as well as of measure 1) that contains no recursive element (Shoenfield [7]), and every $\Sigma_{n}^{0}$ set (i.e., arithmetic set) of positive measure contains an arithmetic element (Sacks [5], and Tanaka [12]). ${ }^{2}$ In view of these results, Hinman [2] asked whether a $\Sigma_{3}^{0}$ set of positive measure must contain a recursive element. The main aim of this note is to give a negative answer for this question; thus, there is a $\Pi_{1}^{0}$ set of positive measure with no recursive element (§1). In $\S 2$, we shall mention some remarks on hierarchy problems.

\section{\$1. Answer for the question.}

LEMma 1. For each positive integer $k$, the measure of every Baire's interval of order $k$ is not greater than $1 / k(k+1)$.

Proof. Let $\left\{a_{1}, \cdots, a_{k}, \cdots\right\}$ be an arbitrary sequence of positive integers. We define $p_{k}=\left[a_{1}, \cdots, a_{k}\right]$ as follows:

$$
\left\{\begin{aligned}
p_{0} & =[\phi]=1, p_{1}=\left[a_{1}\right]=a_{1}, \\
p_{k} & =\left[a_{1}, \cdots, a_{k}\right]=\left[a_{1}, \cdots, a_{k-1}\right] a_{k}+\left[a_{1}, \cdots, a_{k-2}\right] \quad(k \geq 2), \\
& =p_{k-1} a_{k}+p_{k-2}
\end{aligned}\right.
$$

Further, let $q_{0}=0$ and $q_{k}=\left[a_{2}, \cdots, a_{k}\right](k \geq 1)$. Then, by (1), we have

Received October 28, 1968

1) In the present paper, sets means subsets of Baire's zero-space $N^{N}$. Measure means the Lebesgue measure, and we shall write $\mu(E)$ for the measure of a measurable set $E$.

2) An element of Baire's space is regarded as a 1-place number-theoretic function. 


$$
q_{k}=q_{k-1} a_{k}+q_{k-2}(k \geq 2) .
$$

It is well-known by elementary number theory that the following equations hold:

$$
\begin{gathered}
p_{k} q_{k-1}-p_{k-1} q_{k}=(-1)^{k} \quad(k \geq 1), \\
\frac{1 \mid}{\mid a_{1}}+\frac{1 \mid}{\mid a_{2}}+\cdots+\frac{1 \mid}{\mid a_{k}}=\frac{q_{k-1} a_{k}+q_{k-2}}{p_{k-1} a_{k}+p_{k-2}} \text { if } k \geq 2 .
\end{gathered}
$$

Let $\delta=\left\langle a_{1}, \cdots, a_{k}\right\rangle$ be an arbitrary Baire's interval of order $k$. Then by (3) and (4), we have

$$
\begin{aligned}
\mu(\delta) & =\left|\left(\frac{1 \mid}{\mid a_{1}}+\cdots+\frac{1}{\mid a_{k-1}}+\frac{1}{\mid a_{k}}\right)-\left(\frac{1 \mid}{\mid a_{1}}+\cdots+\frac{1}{\mid a_{k-1}}+\frac{1}{\mid a_{k}+1}\right)\right| \\
& =1 /\left(p_{k-1} a_{k}+p_{k-2}\right)\left(p_{k-1} a_{k}+p_{k-1}+p_{k-2}\right) .
\end{aligned}
$$

Since $p_{k} \geq k$ for all $k \geq 1, \mu(\delta) \leqq \frac{1}{(2 k-3)(3 k-4)}$ if $k \geq 2$. Hence we have

$$
\mu(\delta) \leq 1 / k(k+1),
$$

if $k \geq 3$. Obviously (5) holds for $k=1$ or 2 , too.

In the following, a method by which one can evaluate the outermeasure of a countable set is available.

For each numbers $p$ and $e$ we shall define a set $M_{p, e}$ as follows:

$$
\alpha \in M_{p, e} \leftrightarrow(\forall x)_{x<p+e+1}(\exists y)\left[T_{1}(e, x, y) \& \alpha(x)=U(y)\right],
$$

and let

$$
M_{p}=\bigcup_{e=0}^{\infty} M_{p, e}
$$

For each $p$ and $e, M_{p, e}$ is either the empty set or a Baire's interval of order $p+e+1$, and $M_{p}$ is a $\sum_{1}^{0}$ set which contains all recursive elements. By Lemma 1, we have

$$
\mu\left(M_{p}\right) \leq \sum_{e=0}^{\infty} \mu\left(M_{p, e}\right) \leq \sum_{e=0}^{\infty} \frac{1}{(p+e+1)(p+e+2)}=\frac{1}{p+1} .
$$

Thus we obtain the

Theorem 2. There exists a $\sum_{1}^{0}$ set $M\left(\subset N \times N^{N}\right)$ such that each $M_{p}=$ $\{\alpha:\langle p, \alpha\rangle \in M\}$ contains all recursive elements and satisfies the following condition: 


$$
\mu\left(M_{p}\right) \leq \frac{1}{p+1}^{3}
$$

Corollary 3. There exists a $\Pi_{1}^{0}$ set of positive measure that contains no recursive element. ${ }^{3}$ )

This gives a negative answer for Hinman's problem. By a theorem obtained by Sacks [5] and the author [12] (see Introduction), any set obtained in Corollary 3 must contain an arithmetic element.

COROLlary 4. There exists a $\sum_{2}^{0}$ set of measure 1 which has no recursive element.

It follows from Shoenfield-Hinman's Theorem [2; p. 1] (see Introduction) that such a set as in Corollary 4 is an example of arithmetic, meager (first Baire category) sets having measure 1.4),5)

\$2. Some remarks. $1^{\circ}$ Evidently, there is a $\Sigma_{1}^{0}$ set $E$ of measure 1 such that $E \Phi \Re$, where $\Re$ is the set of all 1-place recursive functions.

$2^{\circ}$ ) Contrasting with Corollary 4 , if $E$ is a $\Pi_{2}^{0}$ set of measure 1 then $E$ contains a recursive element. For, since every $\sum_{1}^{0}$ set of measure 1 is an open dense set, $E$ is co-meager (the complement of a meager set) and hence $E$ is not meager. By the Shoenfield-Hinman Theorem, $E$ contains a recursive element.

$3^{\circ}$ ) There is a $\Pi_{1}^{0}$ set consisting of a single element that is not arithmetical. (Spector [10; Corollary 2])

$4^{\circ}$ ) It is known as Kripke-Feferman-Harrison's Theorem (e.g. Mathias [4; $T$ 3200]) that every countable $\sum_{1}^{1}$ set contains only $H A$ elements. This can be proved, for example, by the fact that a non-empty $\Sigma_{1}^{1}$ set with no $H A$ element is dense-in-itself. The elements of a countable $\Sigma_{1}^{1}$ set are not necessarily enumerated by a $H A$ function, as is obvious; but, by the following proposition, the elements of a countable $\triangle_{1}^{1}$ set can be enumerated by a HA function:

3) N. Tsukada has pointed out that this result can be straightforwardly extended in the case of sets of level $|a|$ for $a \in O$.

4) The referee called my attention to this fact.

5) Theorem 2, Corollaries 3 and 4 hold true for the case of the space $2^{N}$ (instead of $\left.N^{N}\right)$. 
Proposition 5. A countable $\Sigma_{1}^{1}$ set $E$ can not contain $H A$ elements of arbitrarily high degrees; that is, there is a $H A$ function $\varphi$ such that

$$
(\forall \alpha)\left[\alpha \in E \rightarrow \alpha \leq_{T} \varphi\right]
$$

Proof. By the Kripke-Feferman-Harrison Theorem, we have

$$
(\forall \beta)(\exists a)\left[\beta \in E \rightarrow a \in O \quad \& \quad \beta \leq_{T} H_{a}\right],
$$

where $\beta \leq_{T} A$ means that $\beta$ is Turing reducible to $A$, namely $\beta$ is recursive in $A$. Since $E$ is $\Sigma_{1}^{1}$, the predicate described in the brackets is $\Pi_{1}^{1}$. Hence, by Kreisel's Lemma [3; Lemma 1], there exists a $H A$ functional $\Psi \in N^{N^{N}}$ such that

$$
(\forall \beta)\left[\beta \in E \rightarrow \Psi\langle\beta\rangle \in O \quad \& \quad \beta \leq_{T} H_{\Psi\langle\beta\rangle}\right] .
$$

The set $\{\Psi\langle\beta\rangle: \beta \in E\}$ is a $\Sigma_{1}^{1}$ subset of $O$. Therefore, by a fact known as a direct consequence of Spector [9; Theorem 1], there exists a number $b \in O$ such that

$$
|\Psi\langle\beta\rangle| \leq|b| \quad \text { for all } \beta \in E \text {. }
$$

Thus we obtain the following implication:

$$
\beta \in E \rightarrow \beta \leq_{T} H_{b} .
$$

This completes the proof.

$5^{\circ}$ ) It is a difficult work that one performs any enumeration of a countable $C A$ (i.e., co-analytic) subset of $N^{N}$. Now one knows MansfieldSolovay's Theorem [11; Appendix II], [4; T3206] and [6]: Let $E$ be a $\sum_{2}^{1}-$ in- $\alpha$ set ( $\alpha$ is a code of $E$ ). If $E$ has a non constructible-from- $\alpha$ element, then $E$ contains a perfect subset. By the theorem, we shall try to do this work for a countable $P C A$ set, thus:

For the sake of simplicity, we shall deal with effective case, i.e., with a countable $\sum_{2}^{1}$ set $E$, instead of a classical PCA set. By the above theorem we have

$$
E \subset L \cap N^{N}
$$

Since $E$ is $\sum_{2}^{1}$, by Shoenfield's Theorem [4;T3101] together with (1) $E$ is a constructible set. Since $\alpha \in L \cap N^{N} \rightarrow \alpha \in F^{6} \boldsymbol{\aleph}_{1}^{L} \subset F^{6} \boldsymbol{\aleph}_{1}$, we have 


$$
E \in L \& E \subset F^{6 \varsigma} \aleph_{1} \& \operatorname{Card}(E)=\aleph_{0} .
$$

(L and F are Gödel's.) Hence by [8; p. 317] we have

$$
E \in F^{\varsigma 6} \aleph_{1} \text {; i.e., } \operatorname{Od}(E)<\aleph_{1} \text {. }
$$

Thus we obtain

Proposition 6.6) Let $E$ be a countable $\Sigma_{2}^{1}$ set. Then $E$ itself is constructible and $\operatorname{Od}(E)<\aleph_{1}$.

Let $\sigma=\operatorname{Od}(E)$. Then $(\forall \beta)[\beta \in E \rightarrow \operatorname{Or}(\beta)<\sigma] .{ }^{7)} \quad$ Note that $\operatorname{Or}(\beta) \leq \operatorname{Od}(\beta)$. Let $\varphi$ be a code for the countable ordinal $\sigma$. We shall inductively define $\alpha$ as follows:

$$
\left\{\begin{array}{l}
\alpha(0)=(\mu i)_{i \in \omega}(\exists \beta)\left[\omega \times \omega \cdot F\left(\varphi_{i}\right)=\beta \& \beta \in E\right], \\
\alpha(n+1)=(\mu i)_{i \in \omega}(\exists \beta)\left[\omega \times \omega \cdot F\left(\varphi_{i}\right)=\beta \quad \& \beta \in E \&(\forall k)_{k \leq n}(i \neq \alpha(k))\right] .
\end{array}\right.
$$

Then we can see that $\alpha$ is $\Delta_{3}^{1}$-in- $\varphi$. Let $\beta_{n}=\omega \times \omega \cdot F\left(\varphi_{\alpha(n)}\right) .\left(\beta_{n}\right.$ is a different notation from $\varphi_{i}$.) Then $E=\left\{\beta_{0}, \beta_{1}, \beta_{2}, \cdots\right\}$. Now, since

$$
\beta_{n}(x)=y \longleftrightarrow(\exists \varepsilon)(\exists \beta)[M(\varphi, \varepsilon) \& A(\varphi, \varepsilon, \beta, \alpha(n)) \& \beta(x)=y],
$$

it is $\sum_{3}^{1}$-in- $\varphi$ and hence $\Delta_{3}^{1}$-in- $\varphi$. Consequently, $E$ can be enumerated by $a$ $\Delta_{3}^{1}$-in- $\varphi$ function. We do not know, however, what $\varphi$ is.

If $\varphi$ is a constructible function (e.g., if $\aleph_{1}^{L}=\aleph_{1}$ then it is the case), then

$(\exists \varphi)\left[\varphi \in L \cap N^{N} \& W(\varphi) \&(\forall \beta)\left[\beta \in E \rightarrow(\exists i)\left[\operatorname{Or}(\beta)<\varphi_{i}\right]\right)\right]$.

Hence we can choose a $\Delta_{3}^{1}$ function $\varphi$ satisfying the bracketed condition. After all, $E$ can be enumerated by a $\Delta_{3}^{1}$ function.

\section{REFERENCES}

[1] J.W. Addison, Some consequences of the axiom of constructibility, Fund. Math., 46 (1959), 337-357.

[2] P.G. Hinman, Some applications of forcing to hierarchy problems in arithmetic, to appear.

[ 3 ] G. Kreisel, The axiom of choice and the class of hyperarithmetic functions, Indag. Math., 24 (1962), 308-319.

[4] A.R.D. Mathias, A survey of recent results in set theory, Mimeographed note, Stanford University, (1968).

6) This proposition is due to $Y$. Sampei.

7) We use freely some results and notations in Addison [1]. 
[5] G.E. Sacks, Measure-theoretic uniformity in recursion theory and set theory, to appear. Summary of results in Bull. Amer. Math. Soc., 73 (1967), 169-174.

[6] Y. Sampei, A proof of Mansfield's Theorem by forcing method, to appear.

[ 7 ] J.R. Shoenfield, The class of recursive functions, Proc. Amer. Math. Soc., 9 (1958), 690-692.

[ 8 ] - Mathematical Logic, Addison-Wesley Company (1967).

[ 9 ] C. Spector, Recursive well-orderings, J. Symbolic Logic, 20 (1955), 151-163.

[10] - , Hyperarithmetical quantifiers, Fund. Math., 48 (1960), 313-320.

[11] R. Solovay, On the cardinality of $\sum_{2}^{1}$ sets of reals, to appear.

[12] H. Tanaka, Some results in the effective descriptive set theory, Publ. RIMS, Kyoto Univ., Ser. A, 3 (1967), 11-52.

Hosei University 